\title{
Hans Rademacher (1892-1969)
}

by

\author{
Bruce C. Berndt (Urbana, Ill.)
}

Hans Rademacher was born of Lutheran parents on April 3, 1892 in Wandsbek, near Hamburg. His mother and father, Emma and Henry, owned a local store. There were two others in the family, a brother Martin and a sister Erna.

By the time Rademacher entered the University in Göttingen in 1910, he had developed a great breadth of interests, an attribute that would be transformed in his later mathematical life into a creative interest in many branches of mathematics. At the age of 18 he had a curiosity for mathematics, the natural sciences, foreign languages, and philosophy. It was to the latter area that Rademacher devoted his primary initial attention. However, he enjoyed the lectures of two of Felix Klein's assistants, Erich Hecke and Hermann Weyl, and eventually he turned to mathematics, chiefly through the influence of Richard Courant. His doctoral dissertation [1] was in real analysis and was completed under the direction of C. Carathéodory in 1916, despite the fact that during the years 1914-1916 he served with his country's army.

Rademacher's first position was as a teacher in Wickersdorf. From 19191922 he served as a Privat Dozent in Berlin. In 1922 he accepted the position of Ausserordentlicher Professor at the University of Hamburg, a position he held until being named Professor at Breslau in 1925. While in Berlin, he married Suzanne Gaspary, and their daughter Karin was born in 1925. The marriage ended in divorce in 1929.

Throughout his entire life, Rademacher maintained an active interest in human rights. In Breslau he joined the International League for the Rights of Men and was chairman of the local chapter of the Deutsche Friedensgesellschaft. Not surprisingly, after Hitler's ascendancy to power, Rademacher was removed from his position in 1934. He and his daughter Karin moved briefly to a small town on the Baltic Sea where he met and married Olga Frey. In that same year 1934, Rademacher departed for the United States, where he had been invited to the University of Pennsylvania as a Visiting Rockefeller Fellow. Rademacher's wife stayed behind in Germany until their 
son Peter was born in 1935. Upon the completion of his fellowship, he returned briefly to Germany but came back in the autumn of 1935 to accept a position at the University of Pennsylvania, the institution he was to serve faithfully until his retirement in 1962. His daughter Karin stayed behind in Germany, and finally in 1947 he succeeded in helping her come to the United States.

Although Rademacher had held a full professorship in Germany for ten years before immigrating to the United States, he was offered only an assistant professorship at Pennsylvania in 1935. Despite this, Rademacher was ever after to remain loyal to the University of Pennsylvania for providing him refuge from the horror that had engulfed his native land. Rademacher enjoyed congenial relations with many colleagues and graduate students at the University of Pennsylvania as well as in Swarthmore where he and his family resided. Among his best friends were A. Zygmund, J. R. Kline, A. Dresden from Swarthmore College, and I. J. Schoenberg, whom he had previously known in Germany (and who recently died on February 21, 1990 at the age of 86). Rademacher's second marriage also ended in divorce in 1947, and he moved to Philadelphia close to the campus of the University of Pennsylvania. In 1949, Rademacher married Schoenberg's sister Irma Wolpe, who had a successful career as a concert pianist. She and her husband opened their home frequently to mathematicians, musicians, and a host of other friends. An annual event at the Rademacher home was an informal New Year's Day afternoon gathering.

During his residence in Swarthmore, Rademacher's good friend A. Dresden introduced him to the Society of Friends. Rademacher joined the Society and remained a member until his death.

In Germany and while at Pennsylvania, Rademacher supervised the Ph.D. dissertations of twenty-one students, many of whom became wellknown mathematicians. These twenty-one doctoral students were Theodor Estermann, Wolfgang Cramer, Otto Schulz, Käthe Silberberg, Albert Whiteman, Joseph Lehner, Lowell Schoenfeld, Ruth Goodman, John Livingood, Paul Bateman, Jean Walton, Nelson Brigham, Emil Grosswald, Saul Rosen, Leila Dragonette, Albert Schild, Jean Calloway, Morris Newman, Frederick Homan, William Spohn, and George Andrews. At the time of this writing, the Rademacher mathematical family tree is blooming into its fifth generation with over 140 flowers.

In 1938, Rademacher and James Clarkson began to conduct a problems seminar at Pennsylvania. Later, after Clarkson departed for service in World War II, Schoenberg took his place. Through the seminar's success over several years, many of the problems became widely known. The seminar was officially listed in the University of Pennsylvania's catalog as the "Proseminar". It was a required course for students seeking a graduate 
degree, and was often used in the determination of a student's progress and the awarding of fellowships.

During the spring semester of 1953, Rademacher held a position at the Institute for Advanced Study in Princeton. In 1954-55, he lectured at the Tata Institute of Fundamental Research in Bombay and at the University of Göttingen. In 1952 and 1959-60, he was Phillips Lecturer at Haverford College, and in 1960-61 he again visited the Institute for Advanced Study. He also had lengthy stays at the University of Oregon and at the University of California at both Berkeley and Los Angeles.

During his final year at Pennsylvania, a special year in the theory of numbers was convened, and several prominent number theorists participated in this tribute to Rademacher. His university awarded him an honorary doctorate upon his retirement in 1962. After retiring from the University of Pennsylvania, Rademacher lectured at New York University for two years. In 1964, he accepted a position at Rockefeller University in New York, a post he held until his death on February 7, 1969. Rademacher became a founding editor of Acta Arithmetica in 1935 and served on its editorial board until his death.

Rademacher made significant contributions to several areas of mathematics, including real analysis and measure theory, complex analysis, number theory, geometry, and numerical analysis. He also coauthored two papers in physics [21], [22] and wrote an article in genetics [31]. About 50 of the 76 papers listed in his bibliography are concerned with number theory or related areas. In this brief survey of Rademacher's achievements, we shall confine ourselves mainly to these 50 articles. Note that there are two bibliographies at the end of this essay. The second (pp. 227-231) comprises the works of Rademacher divided into three categories: papers, books and lecture notes, and problems; the first bibliography contains articles of other authors cited in this survey.

The fourteen papers published by Rademacher in 1916-1922 chiefly concern the theory of functions of a real variable and measure theory. In particular, we mention paper [13] in which Rademacher introduced an orthogonal system of functions now known as Rademacher functions. He soon wrote a sequel on the completion of this system but was discouraged from publishing it. In the meanwhile, J. L. Walsh [59] independently completed the system and published a paper containing much of Rademacher's work. Rademacher never published his paper, which, for many years, was considered to be permanently lost. In the summer of 1979, the paper was miraculously unearthed, and soon thereafter E. Grosswald [16] described the paper's history and its contents at a conference honoring Grosswald at his retirement. Since its discovery, Rademacher's orthonormal system has been utilized in numerous instances in many areas of analysis. 
Papers [3] and [10] might be classified under the purview of number theory as well as analysis. In [3], Rademacher gives an easy proof of a theorem of Borel: The probability that in a decimal expansion not all ten digits appear asymptotically the same number of times is zero. A sharper version of the theorem is proved in [10]. If $n_{\nu}(x)$ denotes the number of times the digit $\nu, 0 \leq \nu \leq 9$, appears in the first $n$ digits of the decimal expansion of a number $x \in(0,1)$, then for any $\varepsilon>0$,

$$
\frac{n_{\nu}(x)}{n}-\frac{1}{10}=O\left(\frac{(\log n)^{1 / 2+\varepsilon}}{n^{1 / 2}}\right),
$$

as $n$ tends to $\infty$, except on a set of measure 0 . Actually, a stronger theorem was previously given by G. H. Hardy and J. E. Littlewood [19], [18, pp. 2863]. Rademacher's proof, however, is short and elegant. It depends upon one of his theorems giving necessary and/or sufficient conditions on the sequence $\left\{e_{n}\right\}$ of complex numbers for the convergence of $\sum u_{n} e_{n}$, where $\sum u_{n}$ is divergent and $u_{n}$ is strictly decreasing to 0 .

Rademacher's first particularly notable contribution to number theory is his improvement of Brun's sieve [14]. As an application of his theorem, Rademacher showed that if $f(x)$ is an irreducible, primitive polynomial of degree $g$, the sequence $\{f(n)\}$ contains infinitely many terms with at most $4 g-1$ prime factors, not counting those which appear in every term of the sequence. For the twin prime problem, it follows from Rademacher's work that there exist infinitely many pairs $n, n+2$ such that each member contains no more than seven prime factors. From Brun's original theorem, this conclusion could be drawn if "seven" were replaced by "nine". In [15], Rademacher showed how Brun's method could be applied to algebraic number fields. For a lucid, contemporary account of Brun's sieve, see Halberstam and Richert's treatise [17, pp. 56-68].

In [16], [17], and [23], Rademacher generalized to algebraic number fields the work of Hardy and Littlewood [20], [18, pp. 561-630] on expressing a positive integer as a sum of three or more primes. Hardy and Littlewood had shown that if there is a number $\theta<3 / 4$ such that no Dirichlet $L$-function has a zero with real part greater than $\theta$, then, subject to an obvious parity condition, there is an asymptotic formula for the number of ways of expressing a positive integer as a sum of $m$ primes, where $m \geq 3$. Rademacher's extension of this result to algebraic number fields requires the existence of a number $\theta^{\prime}<3 / 4$ such that no Hecke zeta-function for the relevant algebraic number field has a zero with real part greater than $\theta^{\prime}$. Just as the results of Hardy and Littlewood [20] were later established by I. M. Vinogradov and A. Walfisz with no unproved hypothesis, in an analogous way, Rademacher's papers [16], [17], and [23] were later superseded by the work of O. Körner [29], [30], [31]. 
The majority of Rademacher's subsequent contributions to number theory came in the related areas of modular forms, partition problems, and Dedekind sums. We first describe a few miscellaneous results.

Rademacher's first research paper in the theory of modular forms is [26]. Using the Schreier-Reidemeister method, he determined a system of generators for the congruence subgroups $\Gamma_{0}(p)$ of the modular group, where $p$ is a prime. He also determined all relations involving the generators. Later, H. Frasch [12] and Grosswald [14] proved similar theorems for the groups $\Gamma(p)$ and $\Gamma_{0}^{0}(p)$, respectively. Grosswald [15] also proved that under certain conditions the generators for $\Gamma(p)$ could be chosen so that none is parabolic. It was not until 1973 that corresponding theorems for $\Gamma_{0}(N)$, where $N$ is an arbitrary positive integer, were established by Y. Chuman [9]. A completely new method, based on Farey symbols, has recently been devised by R. S. Kulkarni [32].

In [65] and [68], Rademacher gives a short, clever proof, based on a corollary of Jensen's inequality, of the following well-known theorem: $A$ modular function analytic and bounded on the open upper half-plane and belonging to a congruence subgroup of the modular group is necessarily a constant.

Recall that the Dedekind eta-function $\eta(\tau)$ is defined for $\operatorname{Im} \tau>0$ by

$$
\eta(\tau)=e^{\pi i \tau / 12} \prod_{n=1}^{\infty}\left(1-e^{2 \pi i n \tau}\right) .
$$

Rademacher devoted several papers to the study of this function and the Dedekind sums which appear in the transformation formulae of $\log \eta(\tau)$. In order to define the Dedekind sum $s(h, k)$, set

$$
((x))= \begin{cases}x-[x]-1 / 2 & \text { if } x \text { is not an integer, } \\ 0 & \text { otherwise. }\end{cases}
$$

Then

$$
s(h, k):=\sum_{\mu=1}^{k}((h \mu / k))((\mu / k)),
$$

where $h$ and $k$ are coprime integers with $k \geq 1$.

Rademacher's first paper devoted to the eta-function and Dedekind sums was published in 1932 [27]. Here he proves the transformation formula for $\log \eta(\tau)$ under modular transformations via contour integration and the functional equation of the Hurwitz zeta function. A similar proof of the transformation formula for $T(\tau)=-1 / \tau$ was later given by A. Weil [61]. One of the most beautiful methods for obtaining the transformation formulae for $\log \eta(\tau)$ is by contour integration and is due to C. L. Siegel [58] and Rademacher [58]. Siegel first gave the proof when $T(\tau)=-1 / \tau$, and then 
Rademacher gave the proof for a general modular transformation. Another general method for obtaining the transformation formulae for $\log \eta(\tau)$ and similar functions has been devised by B. Berndt [4], [5].

Recall that the classical theta-function $\theta_{3}(\tau)$ may be defined by

$$
\theta_{3}(\tau)=\prod_{n=1}^{\infty}\left(1-e^{2 \pi i n \tau}\right)\left(1-e^{\pi i(2 n-1) \tau}\right)^{2},
$$

where $\operatorname{Im} \tau>0$. Since $\theta_{3}$ can be expressed as a quotient of three etafunctions, a transformation formula for $\log \theta_{3}(\tau)$ can be effected from that of the Dedekind eta-function. In one of his last papers [73], Rademacher used this idea to give a new formulation of the transformation formula for $\log \theta_{3}(\tau)$. A similar transformation formula for $\log \theta_{3}(\tau)$ involving a new analogue of Dedekind sums was discovered by Berndt [7].

The most fundamental property of Dedekind sums is the remarkable reciprocity law: If $h, k>0$ and $(h, k)=1$, then

$$
s(h, k)+s(k, h)=-\frac{1}{4}+\frac{1}{12}\left(\frac{h}{k}+\frac{k}{h}+\frac{1}{h k}\right) .
$$

This formula was first proved by R. Dedekind [11] using the transformation formulae of $\log \eta(\tau)$. There now exist many proofs of (1), and several of these can be found in a monograph [7] on Dedekind sums, written by Rademacher and completed by Grosswald after Rademacher's death. Rademacher found five original proofs of (1). In the aforementioned paper [27], Rademacher gives an elegant proof of (1) based on the enumeration of lattice points in pyramids. An elementary proof of (1), as well as a generalization when $(h, k)>1$, is given in [28]. Another elementary proof appears in a paper [44], coauthored with A. L. Whiteman. A method employing finite Fourier series was used in [32] to prove (1). In [53], Rademacher gives a particularly simple and elegant proof of (1) that depends on Riemann-Stieltjes integrals. Rademacher [57] also established the following generalization of the reciprocity law. Let $a, b$, and $c$ denote pairwise coprime, positive integers. Let $a^{\prime}, b^{\prime}$, and $c^{\prime}$ be integers chosen so that $a a^{\prime} \equiv 1(\bmod b c), b b^{\prime} \equiv 1(\bmod c a)$, and $c c^{\prime} \equiv 1(\bmod a b)$. Then

$$
s\left(b c^{\prime}, a\right)+s\left(c a^{\prime}, b\right)+s\left(a b^{\prime}, c\right)=-\frac{1}{4}+\frac{1}{12}\left(\frac{a}{b c}+\frac{b}{c a}+\frac{c}{a b}\right) .
$$

Two of the most important papers in the theory of Dedekind sums are [44] and [59]. Dedekind [11] stated without proof several properties of $s(h, k)$ in a paper that was written in order to fill some gaps in a famous paper of B. Riemann [52], first published in his "Nachlaß". Rademacher and Whiteman [44] gave proofs of these properties and established some others 
as well, including various congruences satisfied by Dedekind sums. We mention a few of the interesting properties established by Rademacher in [59]. If $1<h<k$, then $s(h, k)<s(1, k)$. If $0<h<(k-1)^{1 / 2}$, then $s(h, k)>0$. Rademacher also showed that $12 s(h, k)$ assumes only one integral value, namely 0 , and this occurs if and only if $h \equiv-1(\bmod k)$. Let $\varrho=h / k$ and put $s(\varrho)=s(h, k)$. Then Rademacher showed that $s(\varrho)$ is unbounded from above and from below on any interval in $(-\infty, \infty)$. Identities for $s\left(h_{1}, k_{1}\right) \pm s\left(h_{2}, k_{2}\right)$ are found, where $h_{1} / k_{1}$ and $h_{2} / k_{2}$ are consecutive fractions in a Farey sequence. Rademacher ([8] in problems list) later asked if $s\left(h_{1}, k_{1}\right)+s\left(h_{2}, k_{2}\right)$ is always positive. However, L. Pinzur [46] and K. H. Rosen [53] independently exhibited infinite classes of pairs $\left(h_{1}, k_{1}\right)$, $\left(h_{2}, k_{2}\right)$ for which the sum is negative.

There exists a connection between Dedekind sums and lattice points in a tetrahedron. Let $N_{3}(a, b, c)$ denote the number of lattice points in the tetrahedron

$$
0 \leq x<a, \quad 0 \leq y<b, \quad 0 \leq z<c, \quad 0<x / a+y / b+z / c<1,
$$

where $a, b$, and $c$ are relatively prime in pairs. Rademacher proved the congruence

$$
\begin{aligned}
\left(s(b c, a)-\frac{b c}{12 a}\right)+\left(s(c a, b)-\frac{c a}{12 b}\right) & +\left(s(a b, c)-\frac{a b}{12 c}\right) \\
& \equiv-\frac{1}{4}-\frac{a b c}{12}+\frac{1}{12 a b c}(\bmod 2),
\end{aligned}
$$

and used a theorem of L. J. Mordell [41] to show that

$$
N_{3}(a, b, c) \equiv \frac{1}{4}(a+1)(b+1)(c+1)(\bmod 2) .
$$

More generally, consider the number of lattice points $N_{n}\left(a_{1}, a_{2}, \ldots, a_{n}\right)$ in the $n$-dimensional tetrahedron

$$
0 \leq x_{j}<a_{j}, \quad 1 \leq j \leq n, \quad 0<x_{1} / a_{1}+x_{2} / a_{2}+\ldots+x_{n} / a_{n}<1,
$$

where the integers $a_{j}, 1 \leq j \leq n$, are pairwise coprime. Rademacher ([6] in list of problems) conjectured that

$$
N_{n}\left(a_{1}, a_{2}, \ldots, a_{n}\right) \equiv \frac{1}{2^{n-1}}\left(a_{1}+1\right)\left(a_{2}+1\right) \ldots\left(a_{n}+1\right)(\bmod 2) .
$$

For $n=1,2,(5)$ is trivially true with the congruence sign $\equiv$ replaced by an equality sign. For $n=3,(5)$ is valid by (4). However, for $n=4$, K. H. Rosen [54] found an infinite class of counterexamples to (5).

Very recently, J. E. Pommersheim [48] has removed the coprime conditions on $a, b$, and $c$ and greatly extended the work of Rademacher and Mordell. Moreover, he has also found a beautiful generalization of (2).

Rademacher ([7] in problems list) posed a third problem at the number theory conference at the University of Colorado in 1963. Are the points 
$(h / k, s(h, k))$ dense in the plane? By a very elegant argument using continued fractions, D. R. Hickerson [25] answered this question affirmatively. G. Myerson [42] proved that the sequence $(h / k, s(h, k))$ is uniformly distributed modulo 1, a result further refined by R. W. Bruggeman [8].

Generalizing a sum of F. Klein, Rademacher [70] defined the generalized Dedekind sum

$$
s(h, k ; x, y):=\sum_{\mu=1}^{k}\left(\left(h\left(\frac{\mu+y}{k}+\frac{x}{h}\right)\right)\right)\left(\left(\frac{\mu+y}{k}\right)\right),
$$

where $(h, k)=1$ with $k>0$, and where $x$ and $y$ are real. If $x$ and $y$ are integers, then (6) reduces to $s(h, k)$. Rademacher [70] derived a beautiful reciprocity law relating $s(h, k ; x, y)$ with $s(k, h ; y, x)$.

After Rademacher and Grosswald's elegant monograph [7] on Dedekind sums was published in 1972, there appeared a profusion of papers offering many generalizations. We cannot give any details here, but we remark that many elegant generalizations are due to L. Carlitz. For a survey containing some of these, see Berndt's paper [6]. Analogues or generalizations of the classical Dedekind sum $s(h, k)$ frequently appear in transformation formulae of analogues of $\log \eta(\tau)$, as well as in formulas for values of certain zetafunctions at integral arguments.

Perhaps Rademacher's most famous theorem is his exact formula for the partition function $p(n)$ [37], [38]. Let $c=\pi \sqrt{2 / 3}$ and $\lambda_{n}=\sqrt{n-1 / 24}$. Then Rademacher proved that

$$
p(n)=\frac{1}{\pi \sqrt{2}} \sum_{k=1}^{\infty} A_{k}(n) \sqrt{k} \frac{d}{d n}\left(\frac{\sinh \left(c \lambda_{n} / k\right)}{\lambda_{n}}\right),
$$

where

$$
A_{k}(n)=\sum_{\substack{h(\bmod k) \\(h, k)=1}} \omega_{h, k} e^{-2 \pi i h n / k},
$$

with $\omega_{h, k}=\exp \{\pi i s(h, k)\}$. The history of this formula is interesting.

Introducing their famous circle method, Hardy and Ramanujan [21], [18, pp. 306-339], [50, pp. 276-309] had in 1917 astounded the mathematical community by finding an asymptotic series for $p(n)$, namely,

$$
p(n)=\frac{1}{2 \pi \sqrt{2}} \sum_{k<\alpha \sqrt{n}} A_{k}(n) \sqrt{k} \frac{d}{d n}\left(\frac{e^{c \lambda_{n} / k}}{\lambda_{n}}\right)+O\left(n^{-1 / 4}\right),
$$

as $n$ tends to $\infty$, where $\alpha$ is a positive constant. They started with the familiar generating function $f(x):=\prod_{n=1}^{\infty}\left(1-x^{n}\right)^{-1}$ for $p(n)$, to which they applied Cauchy's integral formula on a circle centered at the origin and interior to the unit circle, and utilized a dissection of that circle by means of 
Farey series. Their method has two main features. First, they approximated $f$ by a function $F$ and integrated $f-F$ over a dissection of the circle by a Farey series of order $N$. Secondly, they coupled the parameters $n$ and $N$ by setting $N=\alpha \sqrt{n}$.

On the other hand, while preparing the lectures for his graduate course in analytic number theory at the University of Pennsylvania in the fall of 1936, Rademacher applied the transformation formula for the Dedekind eta-function $\eta(\tau)=e^{\pi i \tau / 12} / f\left(e^{2 \pi i \tau}\right)$ to obtain the principal term, and he left the parameters $n$ and $N$ free. He subsequently found that

$$
\begin{aligned}
p(n)= & \frac{1}{\pi \sqrt{2}} \sum_{k=1}^{N} A_{k}(n) \sqrt{k} \frac{d}{d n}\left(\frac{\sinh \left(c \lambda_{n} / k\right)}{\lambda_{n}}\right) \\
& +O\left(N^{-1 / 2} \exp \left(2 \pi n N^{-2}\right)\right),
\end{aligned}
$$

as $N$ tends to $\infty$. Note that the principal terms are independent of $N$. Thus, keeping $n$ fixed and letting $N$ tend to $\infty$ in (9), we deduce (7).

Observe that the summands in (7) differ from those in (8). When Rademacher first obtained (7), he assumed that he had made a calculational error. On the following day, Rademacher carefully checked his analysis and found that (7) was indeed correct. D. H. Lehmer [33] later proved that the sum in (8), when extended to $\infty$, diverges.

At about the same time Rademacher proved (7), A. Selberg also proved (7) but never published his result [57, p. 705].

The numbers $A_{k}(n)$ appear in both Hardy and Ramanujan's and Rademacher's formulas, and Lehmer [34] found that they possess some remarkable factorization properties. Using their theorems on congruences for Dedekind sums, Rademacher and Whiteman [44] gave easier proofs of these factorization theorems. Selberg later discovered a much simpler formula for $A_{k}(n)$, namely,

$$
A_{k}(n)=\sqrt{\frac{k}{3}} \sum_{\left(3 j^{2}+j\right) / 2 \equiv-n(\bmod k)}(-1)^{j} \cos \left(\frac{\pi(6 j+1)}{6 k}\right) .
$$

After Whiteman [62] gave the first published proof of (10), Rademacher [61] devised a simpler proof of (10) depending upon the transformation formulae for $\eta(\tau)$. He also gave an improved version of Lehmer's factorization theorems, while offering still easier proofs.

Another interesting theorem is proved in [37]. Let

$$
f(x)=1+\sum_{n=1}^{\infty} p(n) x^{n}, \quad|x|<1 .
$$


Then using (7), Rademacher obtained a representation of the form

$$
f(x)=\sum_{n=0}^{\infty} f_{n}(x),
$$

where each function $f_{n}(x)$ has a simple pole on $|x|=1$; the closure of this set of poles, $|x|=1$, constitutes the natural boundary of $f(x)$. Furthermore, each function $f_{n}(x)$ has an analytic continuation to the entire complex plane. Moreover, (11) represents an analytic function on $|x|>1$, which, from numerical evidence, Rademacher thought might be identically zero. Rademacher ([5, pp. 190-199] in the list of Rademacher's books), in fact, later proved his conjecture. H. Petersson [45] also proved Rademacher's conjecture, but by an entirely different method. J. Lehner [38] established a very general theorem of which the result of Rademacher and Petersson is a special case.

The ideas in Rademacher's paper [38] were found to be even more fruitful, as he and H. S. Zuckerman [40] proved the following generalization: Let $F(\tau)$ be a modular form of dimension $r>0$. Suppose that $F$ has a pole at $i \infty$, i.e.,

$$
F(\tau)=\sum_{n=-\mu}^{\infty} a_{n} e^{2 \pi i(n+\alpha) \tau},
$$

where $\operatorname{Im} \tau>0, \mu>0$, and $0 \leq \alpha<1$. Then the coefficients $a_{n}, n \geq 0$, are determined by those with $-\mu \leq n \leq-1$. Furthermore, Rademacher and Zuckerman derived a formula for $a_{n}, n \geq 0$, which involves $a_{n},-\mu \leq n \leq$ -1 , sums analogous to (10), and the modified Bessel function $I_{r+1}$. An interesting corollary is that an entire modular form of positive dimension belonging to the full modular group must be identically zero. Many other proofs of this fundamental result have been given by various writers. Zuckerman [63] found similar formulas for the coefficients of modular forms associated with congruence subgroups. In a subsequent paper [64], Zuckerman extended the results of himself and Rademacher by establishing a corresponding theorem for modular forms of positive dimension having at most a finite number of poles in a fundamental region. Further extensions to more general automorphic forms were made by J. Lehner [37], [39], [40], who also generalized the aforementioned theorem on identically vanishing forms. For the special cases of the classical theta-functions, L. A. Goldberg [13] discovered easier proofs of Zuckerman's theorems and also derived analogues of the SelbergWhiteman-Rademacher formula (10). Rademacher extended his ideas yet further [39] and found a formula for the Fourier coefficients of the modular invariant $J(\tau)$, a modular form of dimension 0. In fact, Rademacher's formula for the Fourier coefficients of $J(\tau)$ was actually derived earlier by Petersson [44] by an entirely different method. In [41], Rademacher considered 
the converse problem; taking the Fourier expansion of $J(\tau)$ together with his formula for the coefficients, he showed that they define a modular function. The importance of this paper has been made clear by M. Knopp [28], who showed its connection with Eichler cohomology, Poincaré series, and the construction of modular forms.

As previously indicated, the Hardy-Ramanujan-Littlewood circle method is a key ingredient in Rademacher's proof of his formula for $p(n)$. Rademacher later [48] improved this method by replacing the Farey arcs of circles with centers $h / k+i /\left(2 k^{2}\right)$ and radii $1 /\left(2 k^{2}\right)$, where $h / k$ runs through a Farey sequence. These new paths of integration simplify the estimates of certain integrals and clarify how the singularities contribute to the formula.

Rademacher wrote two further papers [42], [45] on $p(n)$. In the first, coauthored with H. S. Zuckerman, proofs are given of two famous identities of Ramanujan:

$$
\sum_{n=0}^{\infty} p(5 n+4) x^{n}=5 \prod_{n=1}^{\infty} \frac{\left(1-x^{5 n}\right)^{5}}{\left(1-x^{n}\right)^{6}}
$$

and

$$
\sum_{n=0}^{\infty} p(7 n+5) x^{n}=7 \prod_{n=1}^{\infty} \frac{\left(1-x^{7 n}\right)^{3}}{\left(1-x^{n}\right)^{4}}+49 x \prod_{n=1}^{\infty} \frac{\left(1-x^{7 n}\right)^{7}}{\left(1-x^{n}\right)^{8}} .
$$

In the second, Rademacher gave further proofs of these identities as well as other results of this sort. In both papers, the theory of modular forms, in particular, the transformation formulae of the Dedekind eta-function, is used. Motivated by Rademacher's ideas, M. Newman [43] established further identities akin to (12) and (13). The identities (12) and (13), respectively, immediately imply the Ramanujan congruences

$$
p(5 n+4) \equiv 0(\bmod 5)
$$

and

$$
p(7 n+5) \equiv 0(\bmod 7) .
$$

The congruences (14) and (15), respectively, are special cases of the following Ramanujan conjectures:

$$
\begin{array}{ll}
\text { If } 24 m \equiv 1\left(\bmod 5^{n}\right), & \text { then } p(m) \equiv 0\left(\bmod 5^{n}\right), \\
\text { if } 24 m \equiv 1\left(\bmod 7^{n}\right), & \text { then } p(m) \equiv 0\left(\bmod 7^{[(n+2) / 2]}\right) .
\end{array}
$$

The conjecture (17) is given in its corrected form; Ramanujan also conjectured a congruence for powers of 11 . Although Ramanujan proved special cases of his three general conjectures, the first complete proofs of (16) and (17) were given by G. N. Watson [60] in 1938. For powers of 11, Ramanujan found proofs for $n=1,2$, and Lehner [35] used Rademacher's 
ideas in [45] to establish the same congruences. Lehner [36] also proved Ramanujan's conjecture for $n=3$ before A. O. L. Atkin [3] first found a complete proof of the corresponding conjecture for powers of 11 in 1967. Ramanujan's unpublished manuscript on congruences for the partition function has recently been reproduced with his "lost notebook" [51]. An excellent survey of Ramanujan's work on congruences has been written by K. G. Ramanathan [49]. The books of M. Knopp [27, Chapters 7, 8] and G. E. Andrews [2, Chapter 10] contain excellent accounts of Ramanujan's congruences for $p(n)$.

In Rademacher's last paper [74] in the area of modular forms, it is shown that $T\left(p^{r}\right)$ is essentially a Chebyshev polynomial in $T(p)$ of the second kind, where $T(n)$ is the Hecke operator, $p$ is a prime, and $r$ is a positive integer. Hecke [23], [24, pp. 644-671] had previously proved that $T\left(p^{r}\right)$ is a polynomial in $T(p)$.

Before leaving modular forms, we describe Rademacher's contribution, peripherally involving modular forms, to a famous problem from complex analysis. Let $f$ be analytic on the unit disc $|z|<1$ with $f^{\prime}(0)=1$. Then there exists a positive constant $\mathcal{L}$ which is the least upper bound of all $r$ such that the range of $f$ always contains a closed disc of radius $r$. The constant $\mathcal{L}$ is called Landau's constant. L. Ahlfors [1] proved that $\mathcal{L} \geq 0.5$, and C. Pommerenke [47] improved this slightly by showing that $\mathcal{L}>0.5$. On the other hand, Rademacher [47] proved that $\mathcal{L} \leq 2 \pi \Gamma(1 / 3) \Gamma(1 / 6)^{-2}=$ $0.54325 \ldots$, which he conjectured to be the true value of $\mathcal{L}$. The exact value of $\mathcal{L}$ is still not known.

In his papers [33], [34], and [36], Rademacher studied the geometrical distribution of prime numbers $\omega$ in the ring of integers of a real quadratic number field. As was the case with his papers [16], [17], and [23], the arguments involve the use of Hecke's Grössencharakteren and the corresponding Dirichlet series. For a given angular region in the $\left(\omega, \omega^{\prime}\right)$ plane, where $\omega^{\prime}$ is the conjugate of $\omega$, Hecke [22], [24, pp. 249-289] had proved earlier that the number of prime numbers in this angular region having norm not exceeding $x$ is asymptotic to $h^{-1} \operatorname{Li}(x)$ times a geometric proportionality factor, where $h$ is the class number of the field and $\operatorname{Li}(x)$ denotes the logarithmic integral. In [33], Rademacher made Hecke's result more precise by proving it with an error term of the type obtained by de la Vallée Poussin for the ordinary prime number theorem. Next, Rademacher [34] applied the results of [33] to obtain an analogous asymptotic formula with an error term for the number of prime numbers in a region of the form $0<\omega \leq Y, 0<\omega^{\prime} \leq Y^{\prime}$, where $Y Y^{\prime}>2$. In [36], he obtained the same result as in [34] by a direct argument not requiring the results of [33].

We now discuss Rademacher's papers on zeta-functions and related areas. 
Applying the Poisson summation formula to

$$
\sum_{n=-\infty}^{\infty}(\alpha+i(n+u))^{-s}, \quad \alpha>0, \quad \operatorname{Re} s>1,
$$

Rademacher [25] elegantly proved the functional equation of the Riemann zeta-function $\zeta(s)$. Using the same notion, he also established the functional equation satisfied by Dirichlet's $L$-functions $L(s, \chi)$. These ideas have been generalized somewhat by W. Schnee [56].

As usual, put

$$
\Lambda(n)= \begin{cases}\log n & \text { if } n \text { is a prime power, } \\ 0 & \text { otherwise. }\end{cases}
$$

Then, by a well-known formula (Davenport [10, p. 109]),

$$
\sum_{n \leq x}^{\prime} \Lambda(n)=x-\lim _{T \rightarrow \infty} \sum_{|\gamma|<T} x^{\varrho} / \varrho-\log (2 \pi)-\frac{1}{2} \log \left(1-x^{-2}\right),
$$

where $\varrho=\beta+i \gamma$ runs through the non-trivial zeros of $\zeta(s)$, and the prime ' on the summation sign indicates that if $x$ is integral, only $\frac{1}{2} \Lambda(x)$ is counted. The infinite series on the right-hand side is closely related to

$$
\sum_{\gamma} \gamma^{-1} \sin (\gamma y), \quad y=\log x .
$$

Rademacher [64] proved that if the Riemann hypothesis is true, (18) has certain jump discontinuities. He felt that by constructing a similar series with the same jumps, some light might be shed on the distribution of zeros of $\zeta(s)$. L. A. Rubel and E. G. Straus [55] showed that this was doubtful by constructing the type of series sought by Rademacher and proving that (18) has the aforementioned discontinuities under a much weaker assumption than the Riemann hypothesis.

In [62], [63], and [67], Rademacher proved a Phragmén-Lindelöf theorem for an infinite strip that has the advantage that, when applied to problems in prime number theory, it is uniform in several parameters. He then gave applications to the growths of $L(s, \chi)$ and the Dedekind zeta-function in the critical strip.

In [35], Rademacher extended the validity of a fascinating identity of F. John [26] involving a periodic function and gave applications to $\zeta(s)$ and Dedekind's zeta-function.

The last research paper on which we shall comment is [76]. Here, Rademacher properly interpreted and established some results stated by Euler on certain divergent series involving the pentagonal numbers $n(3 n-1) / 2$. Moreover, Rademacher extended some of Euler's results. 
Rademacher's expository papers serve not only as excellent introductions to several of his own research interests, but also to broader areas in number theory.

Analytic additive theory in algebraic number fields is examined in [54]. The problems and differences encountered when generalizing the classical theory over the rational field to algebraic number fields are discussed.

Rademacher's address [43] provides an excellent introduction to the partition function $p(n)$, including the Hardy-Littlewood-Ramanujan circle method, the asymptotic formula of Hardy and Ramanujan, and the exact formula of Rademacher. After a prefatory exposition about modular forms, Rademacher further discusses the content of his recent work [39]-[42].

Rademacher did not deem function theory to be merely a tool in studying properties of numbers. "It is more the inner harmony of a system" with both disciplines bearing on the character of the other. This belief is fully developed by Rademacher in [46]. "The name 'analytic number theory' implies, as I take it, a thorough fusion of analysis and arithmetic, in which as we shall see, analysis is not necessarily subordinate to arithmetic." Often analytic number theory is regarded as the development of asymptotic formulas. However, Rademacher believed that identities, group-theoretical arguments, and structural considerations have just as important roles in the subject. Rademacher provides a brief, eloquent history of analytic number theory in [46], wherein the names of Dirichlet, Riemann, Landau, Hardy, Littlewood, Ramanujan, and many others are highlighted. We must now add to this roll of honor the name of Rademacher.

The author is very grateful to Paul Bateman, Heini Halberstam, Marvin Knopp, Joseph Lehner, and Andrzej Schinzel for many helpful contributions.

\section{References}

[1] L. V. Ahlfors, An extension of Schwarz's lemma, Trans. Amer. Math. Soc. 43 (1938), 359-364.

[2] G. E. Andrews, The Theory of Partitions, Addison-Wesley, Reading, Mass., 1976.

[3] A. O. L. Atkin, Proof of a conjecture of Ramanujan, Glasgow Math. J. 8 (1967), $67-78$.

[4] B. C. Berndt, Generalized Dedekind eta-functions and generalized Dedekind sums, Trans. Amer. Math. Soc. 178 (1973), 495-508.

[5] —, Generalized Eisenstein series and modified Dedekind sums, J. Reine Angew. Math. 272 (1975), 182-193.

[6] - Reciprocity theorems for Dedekind sums and generalizations, Adv. in Math. 23 (1977), 285-316.

[7] -, Analytic Eisenstein series, theta-functions, and series relations in the spirit of Ramanujan, J. Reine Angew. Math. 304 (1978), 332-365.

[8] R. W. Bruggeman, Dedekind sums and Fourier coefficients of modular forms, J. Number Theory 36 (1990), 289-321. 
[9] Y. Chuman, Generators and relations of $\Gamma_{0}(N)$, J. Math. Kyoto Univ. 13 (1973), 381-390.

[10] H. Daven port, Multiplicative Number Theory, 2nd ed., Springer, New York 1980.

[11] R. Dedekind, Erläuterungen zu zwei Fragmenten von Riemann, in: Gesammelte Mathematische Werke, Friedr. Vieweg \& Sohn, Braunschweig 1930, 159-172.

[12] H. Frasch, Die Erzeugenden der Hauptkongruenzgruppen für Primzahlstufen, Math. Ann. 108 (1933), 229-252.

[13] L. A. Goldberg, Transformations of Theta-functions and Analogues of Dedekind Sums, Ph.D. Dissertation, University of Illinois at Urbana-Champaign, 1981.

[14] E. Grosswald, On the structure of some subgroups of the modular group, Amer. J. Math. 72 (1950), 809-834.

[15] - On the parabolic generators of the principal congruence subgroups of the modular group, ibid. 74 (1952), 435-443.

[16] - An orthonormal system and its Lebesgue constants, in: Analytic Number Theory, M. I. Knopp (ed.), Lecture Notes in Math. 899, Springer, Berlin 1981, 2-9.

[17] H. Halberstam and H.-E. Richert, Sieve Methods, Academic Press, New York 1974.

[18] G. H. Hardy, Collected Papers, Vol. 1, Clarendon Press, Oxford 1966.

[19] G. H. Hardy and J. E. Littlewood, Some problems of Diophantine approximation. I. The fractional part of $n^{k} \theta$, Acta Math. 37 (1914), 155-191.

[20] - - - Some problems of 'Partitio Numerorum'; III: On the expression of a number as a sum of primes, ibid. 44 (1922), 1-70.

[21] G. H. Hardy and S. Ramanujan, Asymptotic formulae in combinatory analysis, Proc. London Math. Soc. (2) 17 (1918), 75-115.

[22] E. Hecke, Eine neue Art von Zetafunktionen und ihre Beziehungen zur Verteilung der Primzahlen. Zweite Mitteilung, Math. Z. 6 (1920), 11-51.

[23] —, Über Modulfunktionen und die Dirichletschen Reihen mit Eulerscher Produktenwicklung. I, Math. Ann. 114 (1937), 1-28.

[24] —, Mathematische Werke, Vandenhoeck \& Ruprecht, Göttingen 1970.

[25] D. R. Hickerson, Continued fractions and density results for Dedekind sums, J. Reine Angew. Math. 290 (1977), 113-116.

[26] F. John, Identitäten zwischen dem Integral einer willkürlichen Funktion und unendlichen Reihen, Math. Ann. 110 (1935), 718-721.

[27] M. Knopp, Modular Functions in Analytic Number Theory, Markham, Chicago 1970.

[28] —, Rademacher on $J(\tau)$, Poincaré series of nonpositive weights and the Eichler cohomology, Notices Amer. Math. Soc. 37 (1990), 385-393.

[29] O. Körner, Übertragung des Goldbach-Vinogradovschen Satzes auf reell-quadratische Zahlkörper, Math. Ann. 141 (1960), 343-366.

[30] - Erweiterter Goldbach-Vinogradovscher Satz in beliebigen algebraischen Zahlkörpern, ibid. 143 (1961), 344-378.

[31] —, Zur additiven Primzahltheorie algebraischer Zahlkörper, ibid. 144 (1961), 97109.

[32] R. S. Kulkarni, An arithmetic-geometric method in the study of the subgroups of the modular group, Amer. J. Math. 113 (1991), 1053-1133.

[33] D. H. Lehmer, The Hardy-Ramanujan series for the partition function, J. London Math. Soc. 12 (1937), 171-176.

[34] - On the series for the partition function, Trans. Amer. Math. Soc. 43 (1938), 271-295. 
[35] J. Lehner, Ramanujan identities involving the partition function for the moduli $11^{a}$, Amer. J. Math. 65 (1943), 492-520.

[36] - Proof of Ramanujan's partition congruence for the modulus $11^{3}$, Proc. Amer. Math. Soc. 1 (1950), 172-181.

[37] - , The Fourier coefficients of automorphic forms belonging to a class of horocyclic groups, Michigan Math. J. 4 (1957), 265-279.

[38] -, Partial fraction decompositions and expansions of zero, Trans. Amer. Math. Soc. 87 (1958), 130-143.

[39] - , The Fourier coefficients of automorphic forms on horocyclic groups, II, Michigan Math. J. 6 (1959), 173-193.

[40] - The Fourier coefficients of automorphic forms on horocyclic groups, III, ibid. 7 (1960), 65-74.

[41] L. J. Mordell, Lattice points in a tetrahedron and generalized Dedekind sums, J. Indian Math. Soc. 15 (1951), 41-46.

[42] G. Myerson, Dedekind sums and uniform distribution, J. Number Theory 28 (1988), 233-239.

[43] M. Newman, Remarks on some modular identities, Trans. Amer. Math. Soc. 73 (1952), 313-320.

[44] H. Petersson, Über die Entwicklungskoeffizienten der automorphen Formen, Acta Math. 58 (1932), 169-215.

[45] - Die linearen Relationen zwischen den ganzen Poincaréschen Reihen von reeller Dimension zur Modulgruppe, Abh. Math. Sem. Univ. Hamburg 12 (1938), 415-472.

[46] L. Pinzur, On a question of Rademacher concerning Dedekind sums, Proc. Amer. Math. Soc. 61 (1976), 11-15.

[47] C. Pommerenke, On Bloch functions, J. London Math. Soc. (2) 2 (1970), 689-695.

[48] J. E. Pommersheim, Lattice points in a tetrahedron and toric varieties; Dedekind sum relations and toric varieties, submitted for publication.

[49] K. G. Ramanathan, Ramanujan and the congruence properties of partitions, Proc. Indian Acad. Sci. (Math. Sci.) 89 (1980), 133-157.

[50] S. Ramanujan, Collected Papers, Chelsea, New York 1962.

[51] - , The Lost Notebook and Other Unpublished Papers, Narosa, New Delhi 1988.

[52] B. Riemann, Fragmente über die Grenzfälle der elliptischen Modulfunktionen, in: Gesammelte Mathematische Werke, Dover, New York 1953, 455-465.

[53] K. H. Rosen, On the sign of some Dedekind sums, J. Number Theory 9 (1977), 209-212.

[54] - Lattice points in four-dimensional tetrahedra and a conjecture of Rademacher, J. Reine Angew. Math. 307/308 (1979), 264-275.

[55] L. A. Rubel and E. G. Straus, Special trigonometric series and the Riemann hypothesis, Math. Scand. 18 (1966), 35-44.

[56] W. Schnee, Die Funktionalgleichung der Zetafunktion und der Dirichletschen Reihen mit periodischen Koeffizienten, Math. Z. 31 (1930), 378-390.

[57] A. Selberg, Reflections around the Ramanujan centenary, in: Collected Papers, Vol. 1, Springer, Berlin 1989, 695-706.

[58] C. L. Siegel, A simple proof of $\eta(-1 / \tau)=\eta(\tau) \sqrt{\tau / i}$, Mathematika 1 (1954), 4.

[59] J. L. Walsh, A closed set of normal, orthogonal functions, Amer. J. Math. 55 (1923), 5-24.

[60] G. N. Watson, Ramanujans Vermutung über Zerfällungsanzahlen, J. Reine Angew. Math. 179 (1938), 97-128.

[61] A. Weil, Sur une formule classique, J. Math. Soc. Japan 20 (1968), 400-402. 
[62] A. Whiteman, A sum connected with the series for the partition function, Pacific J. Math. 6 (1956), 159-176.

[63] H. S. Zuckerman, On the coefficients of certain modular forms belonging to subgroups of the modular group, Trans. Amer. Math. Soc. 45 (1939), 298-321.

[64] - On the expansions of certain modular forms of positive dimension, Amer. J. Math. 62 (1940), 127-152.

DEPARTMENT OF MATHEMATICS

UNIVERSITY OF ILLINOIS

1409 WEST GREEN STREET

URBANA, ILLINOIS 61801, U.S.A. 


\section{Publications of Hans Rademacher}

\section{Papers}

[1] Eineindeutige Abbildungen und Messbarkeit, Monatsh. Math. Phys. 27 (1916), 183291.

[2] (with C. Carathéodory) Über die Eineindeutigkeit im Kleinen und im Grossen stetiger Abbildungen von Gebieten, Arch. Math. Phys. (3) 26 (1917), 1-9.

[3] Zu dem Borelschen Satz über die asymptotische Verteilung der Ziffern in Dezimalbrüchen, Math. Z. 2 (1918), 306-311.

[3a] Nachträgliche Bemerkung zu meiner Arbeit "Über die asymptotische Verteilung der Ziffern in Dezimalbrüchen", ibid. 3 (1919), 317.

[4] Über partielle und totale Differenzierbarkeit von Funktionen mehrerer Variabeln und über die Transformation der Doppelintegrale, Math. Ann. 79 (1919), 340-359.

[5] Über streckentreue und winkeltreue Abbildung, Math. Z. 4 (1919), 131-138.

[6] Bemerkungen zu den Cauchy-Riemannschen Differentialgleichungen und zum Moreraschen Satz, ibid. 177-185.

[7] Über partielle und totale Differenzierbarkeit von Funktionen mehrerer Variabeln. II, Math. Ann. 81 (1920), 52-63.

[8] Zur Theorie der Minkowskischen Stützebenenfunktion, S.-B. Berlin. Math. Gesell. 20 (1921), 14-19.

[9] Über eine Eigenschaft von messbaren Mengen positiven Masses, Jber. Deutsch. Math.-Verein. 30 (1921), 130-132.

[10] Über die asymptotische Verteilung gewisser konvergenzerzeugender Faktoren, Math. Z. 11 (1921), 276-288.

[11] Über den Konvergenzbereich der Eulerschen Reihentransformation, S.-B. Berlin. Math. Gesell. 21 (1922), 16-24.

[12] Über eine funktionale Ungleichung in der Theorie der konvexen Körper, Math. Z. 13 (1922), 18-27.

[13] Einige Sätze über Reihen von allgemeinen Orthogonalfunktionen, Math. Ann. 87 (1922), 112-138.

[14] Beiträge zur Viggo Brunschen Methode in der Zahlentheorie, Abh. Math. Sem. Univ. Hamburg 3 (1923), 12-30.

[15] Über die Anwendung der Viggo Brunschen Methode auf die Theorie der algebraischen Zahlkörper, S.-B. Preuss. Akad. Wiss. Phys.-Math. Kl. 24 (1923), 211-218.

[16] Zur additiven Primzahltheorie algebraischer Zahlkörper. I. Über die Darstellung totalpositiver Zahlen als Summe von totalpositiven Primzahlen im reell-quadratischen Zahlkörper, Abh. Math. Sem. Univ. Hamburg 3 (1924), 109-163.

[17] Zur additiven Primzahltheorie algebraischer Zahlkörper. II. Über die Darstellung von Körperzahlen als Summe von Primzahlen im imaginär-quadratischen Zahlkörper, ibid. 331-378. 
[18] Über den Vektorenbereich eines konvexen ebenen Bereiches, Jber. Deutsch. Math.Verein. 34 (1925), 64-79.

[19] Über eine Erweiterung des Goldbachschen Problems, Math. Z. 25 (1926), 627-657.

[20] Eine Bemerkung zu Herrn Peterssons Arbeit: "Über die Darstellung natürlicher Zahlen durch definite und indefinite quadratische Formen von $2 r$ Variablen", Abh. Math. Sem. Univ. Hamburg 5 (1926), 40-44.

[21] (with F. Reiche) Die Quantelung des symmetrischen Kreisels nach Schrödingers Undulationsmechanik, Z. Phys. 39 (1926), 444-464.

[22] (with F. Reiche) Die Quantelung des symmetrischen Kreisels nach Schrödingers Undulationsmechanik. II. Intensitätsfragen, ibid. 41 (1927), 453-492.

[23] Zur additiven Primzahltheorie algebraischer Zahlkörper. III. Über die Darstellung totalpositiver Zahlen als Summen von totalpositiven Primzahlen in einem beliebigen Zahlkörper, Math. Z. 27 (1928), 321-426.

[24] Zur Theorie der Modulfunktionen, in: Atti del Congresso Internazionale dei Matematici, Bologna 1928, Vol. 3, Nicola Zanichelli, Bologna 1928, 297-301.

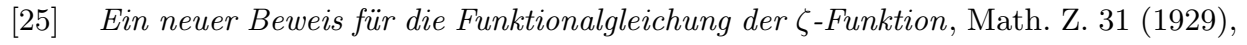
39-44.

[26] Über die Erzeugenden von Kongruenzuntergruppen der Modulgruppe, Abh. Math. Sem. Univ. Hamburg 7 (1929), 134-148.

[27] Zur Theorie der Modulfunktionen, J. Reine Angew. Math. 167 (1932), 312-336.

[28] Eine arithmetische Summenformel, Monatsh. Math. Phys. 39 (1932), 221-228.

[29] Bestimmung einer gewissen Einheitswurzel in der Theorie der Modulfunktionen, J. London Math. Soc. 7 (1932), 14-19.

[30] Lebensbild von Wilhelm Foerster, Schlesische Lebensbilder 3 (1932), 343-348.

[31] Mathematische Theorie der Genkoppelung unter Berücksichtigung der Interferenz, Schlesische Gesell. Vaterländische Cultur (1932), 1-8.

[32] Egy Reciprocitásképletröl a Modulfüggévenyek Elméletéböl, Mat. Fiz. Lapok 40 (1933), 24-34.

[33] Primzahlen reell-quadratischer Zahlkörper in Winkelräumen, Math. Ann. 111 (1935), 209-228.

[34] Über die Anzahl der Primzahlen eines reell-quadratischen Zahlkörpers, deren Konjugierte unterhalb gegebener Grenzen liegen, Acta Arith. 1 (1935), 67-77.

[35] Some remarks on F. John's identity, Amer. J. Math. 58 (1936), 169-176.

[36] On prime numbers of real quadratic fields in rectangles, Trans. Amer. Math. Soc. 39 (1936), 380-398.

[37] A convergent series for the partition function $p(n)$, Proc. Nat. Acad. Sci. U.S.A. 23 (1937), 78-84.

[38] On the partition function $p(n)$, Proc. London Math. Soc. (2) 43 (1937), 241-254.

[39] The Fourier coefficients of the modular invariant $J(\tau)$, Amer. J. Math. 60 (1938), 501-512.

[40] (with H. S. Zuckerman) On the Fourier coefficients of certain modular forms of positive dimension, Ann. of Math. 39 (1938), 433-462.

[41] The Fourier series and the functional equation of the absolute modular invariant $J(\tau)$, Amer. J. Math. 61 (1939), 237-248.

[41a] Correction, ibid. 64 (1942), 456.

[42] (with H. S. Zuckerman) A new proof of two of Ramanujan's identities, Ann. of Math. 40 (1939), 473-489.

[43] Fourier expansions of modular forms and problems of partition, Bull. Amer. Math. Soc. 46 (1940), 59-73. 
[44] (with A. Whiteman) Theorems on Dedekind sums, Amer. J. Math. 63 (1941), 377-407.

[45] The Ramanujan identities under modular substitutions, Trans. Amer. Math. Soc. 51 (1942), 609-636.

[46] Trends in research: the analytic number theory, Bull. Amer. Math. Soc. 48 (1942), 379-401.

[47] On the Bloch-Landau constant, Amer. J. Math. 65 (1943), 387-390.

[48] On the expansion of the partition function in a series, Ann. of Math. 44 (2) (1943), 416-422.

[49] (with I. J. Schoenberg) An iteration method for calculation with Laurent series, Quart. Appl. Math. 4 (1946), 142-159.

[50] On the accumulation of errors in processes of integration on high-speed calculating machines, in: Proceedings of a Symposium on Large-scale Digital Calculating Machinery, Annals of the Computation Laboratory of Harvard University, Vol. 16, Harvard Univ. Press, Cambridge, Mass., 1948, 176-187.

[51] On a theorem of Frobenius, in: Studies and Essays Presented to R. Courant on His 60th Birthday, January 8, 1948, Interscience, New York 1948, 301-305.

[52] (with I. J. Schoenberg) Helly's theorems on convex domains and Tchebycheff's approximation problem, Canad. J. Math. 2 (1950), 245-256.

[53] Die Reziprozitätsformel für Dedekindsche Summen, Acta Sci. Math. (Szeged) 12, Part B (1950), 57-60.

[54] Additive algebraic number theory, in: Proceedings of the International Congress of Mathematicians, Cambridge, Mass., 1950, Vol. 1, Amer. Math. Soc., Providence 1952, 356-362.

[55] On the condition of Riemann integrability, Amer. Math. Monthly 61 (1954), 1-8.

[56] On Dedekind sums and lattice points in a tetrahedron, in: Studies in Mathematics and Mechanics Presented to Richard von Mises, Academic Press, New York 1954, 49-53.

[57] Generalization of the reciprocity formula for Dedekind sums, Duke Math. J. 21 (1954), 391-397.

[58] On the transformation of $\log \eta(\tau)$, J. Indian Math. Soc. 19 (1955), 25-30.

[59] Zur Theorie der Dedekindschen Summen, Math. Z. 63 (1956), 445-463.

[60] Fourier analysis in number theory, Final Technical Report, Symposium on Harmonic Analysis and Related Integral Transforms, Cornell Univ., Ithaca, New York 1956, 25 pages.

[61] On the Selberg formula for $A_{k}(n)$, J. Indian Math. Soc. 21 (1957), 41-55.

[62] On the Phragmén-Lindelöf theorem and subharmonic functions, Technical Report, Cornell Univ., Ithaca, New York 1957, 10 pages.

[63] On the Phragmén-Lindelöf theorem and some applications, in: Seminars on Analytic Functions, Vol. 1, Institute for Advanced Study, Princeton 1958, 314-322.

[64] Remarks concerning the Riemann-von Mangoldt formula, Report of the Institute in the Theory of Numbers, Univ. of Colorado, Boulder, Colorado, 1959, 31-37.

[65] A fundamental theorem in the theory of modular functions, Report of the Institute in the Theory of Numbers, Univ. of Colorado, Boulder, Colorado, 1959, 71-72.

[66] On the Hurwitz zetafunction, Report of the Institute in the Theory of Numbers, Univ. of Colorado, Boulder, Colorado, 1959, 73-77.

[67] On the Phragmén-Lindelöf theorem and some applications, Math. Z. 72 (1959/60), 192-204.

[68] A proof of a theorem on modular functions, Amer. J. Math. 82 (1960), 338-340. 
[69] On a theorem of Besicovitch, in: Studies in Mathematical Analysis and Related Topics, Stanford Univ. Press, Stanford, California, 1962, 294-296.

[70] Some remarks on certain generalized Dedekind sums, Acta Arith. 9 (1964), 97-105.

[71] On the number of certain types of polyhedra, Illinois J. Math. 9 (1965), 361-380.

[72] (with R. Dickson and M. Plotkin) A packing problem for parallelepipeds, J. Combin. Theory 1 (1966), 3-14.

[73] Über die Transformation der Logarithmen der Thetafunktionen, Math. Ann. 168 (1967), 142-148.

[74] Eine Bemerkung über die Heckeschen Operatoren T(n), Abh. Math. Sem. Univ. Hamburg 31 (1967), 149-151.

[75] On the roundest oval, Trans. New York Acad. Sci. (2) 29 (1967), 868-874.

[76] Comments on Euler's "De mirabilibus proprietatibus numerorum pentagonalium", in: Abhandlungen aus Zahlentheorie und Analysis, P. Turán (ed.), Deutscher Verlag Wiss., Berlin 1968, 257-268.

\section{Books and lecture notes}

[1] (with O. Toeplitz) Von Zahlen und Figuren, J. Springer, Berlin 1930; English edition, The Enjoyment of Mathematics, transl. by H. S. Zuckerman, Princeton University Press, Princeton 1957.

[2] (with E. Steinitz) Vorlesungen über die Theorie der Polyeder unter Einschluss der Elemente der Topologie, Grundlehren Math. Wiss. 41, J. Springer, Berlin 1934.

[3] Lecture Notes on Elementary Mathematics from an Advanced Viewpoint, Eugene, Oregon, 1954.

[4] Lecture Notes on Analytic Additive Number Theory, Eugene, Oregon, 1954.

[5] Lectures on Analytic Number Theory, Tata Inst. of Fundamental Research, Bombay $1954 / 55$.

[6] Lectures on Elementary Number Theory, Blaisdell, New York 1964.

[7] (with E. Grosswald) Dedekind Sums, Carus Math. Monograph 16, Math. Assoc. Amer., Washington, D.C., 1972.

[8] Topics in Analytic Number Theory, Grundlehren Math. Wiss. 169, Springer, New York 1973.

[9] Collected Papers of Hans Rademacher (2 volumes), The MIT Press, Cambridge, Mass., 1974.

[10] Higher Mathematics from an Elementary Point of View, D. Goldfeld (ed.), Birkhäuser, Boston 1982.

\section{Problems and solutions to problems}

[1] Aufgabe 26, Jber. Deutsch. Math.-Verein. 34 (1925-26), 98; solutions by M. Krafft and G. Szegö, Jber. Deutsch. Math.-Verein. 35 (1926), 50-52.

[2] Aufgabe 30, Jber. Deutsch. Math.-Verein. 34 (1925-26), 158; solution by A. Brauer, Jber. Deutsch. Math.-Verein. 35 (1926), 92-94.

[3] Aufgabe 31, Jber. Deutsch. Math.-Verein. 34 (1925-26), 158; solution by A. Brauer, Jber. Deutsch. Math.-Verein. 35 (1926), 94-95. 
[4] Aufgabe 32, Jber. Deutsch. Math.-Verein. 34 (1925-26), 158-159; solution by A. Brauer, Jber. Deutsch. Math.-Verein. 35 (1926), 95-96.

[5] Solution to Aufgabe 184 posed by T. Nagell, Jber. Deutsch. Math.-Verein. 46 (1936), 4-5.

[6] Problem 98. Number of lattice points in a tetrahedron, Proceedings of the 1963 Number Theory Conference, Univ. of Colorado, Boulder, Colorado, 1963, 111-112.

[7] Problem 99. Density of the points $(h / k, s(h, k))$, Proceedings of the 1963 Number Theory Conference, Univ. of Colorado, Boulder, Colorado, 1963, 112.

[8] Problem 100. Positivity of Dedekind sums, Proceedings of the 1963 Number Theory Conference, Univ. of Colorado, Boulder, Colorado, 1963, 112. 